Access to this work was provided by the University of Maryland, Baltimore County (UMBC)

ScholarWorks@UMBC digital repository on the Maryland Shared Open Access (MD-SOAR) platform.

Please provide feedback

Please support the ScholarWorks@UMBC repository by emailing scholarworks-group@umbc.edu and telling us what having access to this work means to you and why it's important to you. Thank you. 


\title{
A Partial Optimization Approach for Privacy Preserving Frequent Itemset Mining
}

\author{
Shibnath Mukherjee, Yahoo! Research and Development, India \\ Aryya Gangopadhyay, University of Maryland Baltimore County, USA \\ Zhiyuan Chen, University of Maryland Baltimore County, USA
}

\begin{abstract}
While data mining has been widely acclaimed as a technology that can bring potential benefits to organizations, such efforts may be negatively impacted by the possibility of discovering sensitive patterns, particularly in patient data. In this article the authors present an approach to identify the optimal set of transactions that, if sanitized, would result in hiding sensitive patterns while reducing the accidental hiding of legitimate patterns and the damage done to the database as much as possible. Their methodology allows the user to adjust their preference on the weights assigned to benefits in terms of the number of restrictive patterns hidden, cost in terms of the number of legitimate patterns hidden, and damage to the database in terms of the difference between marginal frequencies of items for the original and sanitized databases. Most approaches in solving the given problem found in literature are all-heuristic based without formal treatment for optimality. While in a few work, ILP has been used previously as a formal optimization approach, the novelty of this method is the extremely low cost-complexity model in contrast to the others. They implement our methodology in $C$ and $C++$ and ran several experiments with synthetic data generated with the IBM synthetic data generator. The experiments show excellent results when compared to those in the literature.
\end{abstract}

Keywords: $\quad$ Data Mining, Data Sanitization, ILP, Optimization, Privacy

\section{INTRODUCTION}

Knowledge discovery from databases (KDD) and knowledge hiding in databases (KHD) are perhaps oxymoron, yet this is indeed the problem faced by the data mining research community these days. Over the past decade, research in the line was focused primarily in discovery

DOI: $10.4018 /$ jcmam.2010072002 of memory efficient and fast algorithms that could discover patterns in large databases in forms of association rules, classification models and clusters of data values. Today with rigorous improvements in the field of these algorithms (Han \& Kamber 2006), the primary area has taken quite a leap forward, but has posed some grave problems as well in terms of security and privacy preservation in the knowledge discovery tasks (Dasseni et al., 2001; Evfimienski et al., 
2002; Oliviera et al., 2003a, 2003b; Han et al., 2006). A number of cases have been reported in literature where data mining actually has posed threats to discovery of sensitive knowledge and violating privacy. One typical problem is that of inferencing, which means inferring sensitive information from non-sensitive or unclassified data (Oliviera et al., 2002; Clifton, 2001).

Data mining is part of the larger business intelligence initiatives that are taking place in organizations across government and industry sectors, many of which include medical applications. It is being used for prediction as well knowledge discovery that can lead to cost reduction, business expansion, and detection of fraud or wastage of resources, among other things. With its many benefits, data mining has given rise to increasingly complex and controversial privacy issues. For example, the privacy implications of data mining have lead to high profile controversies involving the use of data mining tools and techniques on data related to drug prescriptions. Two major health care data publishers filed a petition to the Supreme Court on whether commercial use of data mining is protected by the First Amendment ${ }^{1}$, an appeal to a controversial ruling by the $1^{\text {st }}$ U.S. Circuit Court of Appeals that upheld a 2006 New Hampshire law that banned the usage of doctor's prescription history to increase drug sales.

Privacy implications are a major roadblock to information sharing across organizations. For example, sharing inventory data might reveal information that can be used to gain strategic advantages by competitors. Unless the actual or perceived implications of data mining methods on privacy issues are properly dealt with, it can lead to sub-optimal decision making in organizations, and reluctance to accept such tools by the public in general. For example there could be benefits in sharing prescription data from different pharmacy stores to mine for information such as the use of generic drugs, socio-demographic and geographic analysis of prescription drugs, which will require moving the data from each store or site to a central location, which increases the risks of litigation. In general several potential problems that have been identified for privacy protection make the case for privacy reserving data mining. These include: legal requirements for protecting data (e.g. HIPAA healthcare regulations in the US) Federal register (2002), liability from inadvertent disclosure of data, risk of misuse of proprietary information (Atallah et al., 2003), and antitrust concerns (Vaidya et al., 2006).

Thus it is of growing importance to devise efficient tradeoffs between knowledge discovery and knowledge hiding from databases so that cost to the involved, in general, gets minimized in the process yet the benefit is maximized. The work that will be presented in this article will focus on formulating a model for sanitization of databases against discovery of restrictive associative patterns, while distorting the databases and legitimate pattern discovery as little as possible. To illustrate the problem, consider a classic example given in (Evfimienski et al., 2002; Oliviera et al., 2002). There is a server and several clients, each having its own set of items. The clients want the server to provide them with recommendations based on statistical information about association among items. However the clients do not want the server to know some restrictive patterns. Now what is sent to the server is the raw database and in its process of searching for frequent patterns the server will discover the restrictive patterns as well. Thus what the client has to send is the raw database, modified in a manner so that the restrictive patterns are not discovered. But this needs distortion to the raw database before sending it to the server and the distortion should be such that it is minimal and hiding of the legitimate patterns is also minimal. Other examples of the problem are given in (Verikyos et al., 2004). The example shows the vulnerability of critical frequent patterns, however it is directly associated with the problems of exposing critical association rules as well since rules are built from patterns. Indeed some of the research work like (Verikyos et al 2004) use reduction of support of sensitive frequent patterns as one of the methods to hide association rules that could be generated from them. All these methods are based on modifying the 
13 more pages are available in the full version of this document, which may be purchased using the "Add to Cart" button on the publisher's webpage:

www.igi-global.com/article/partial-optimization-approachprivacy-preserving/38942

\section{Related Content}

Mining Association Rules from Fuzzy DataCubes

Nicolás Marín, Carlos Molina, Daniel Sánchez and M. Amparo Vila (2010). Scalable Fuzzy Algorithms for Data Management and Analysis: Methods and Design (pp. 84129).

www.irma-international.org/chapter/mining-association-rules-fuzzydatacubes/38566/

Performance Enhancement of Differential Evolution by Incorporating Lévy Flight and Chaotic Sequence for the Cases of Satellite Images

Krishna Gopal Dhal, Md. Iqbal Quraishi and Sanjoy Das (2015). International Journal of Applied Metaheuristic Computing (pp. 69-81).

www.irma-international.org/article/performance-enhancement-of-differentialevolution-by-incorporating-Ivy-flight-and-chaotic-sequence-for-the-cases-ofsatellite-images/129012/

Exterior Path Relinking for Zero-One Optimization

Fred Glover (2014). International Journal of Applied Metaheuristic Computing (pp. 1 8).

www.irma-international.org/article/exterior-path-relinking-for-zero-oneoptimization/117263/

Improving Switched Current Sigma Delta Modulators' Performances via the Particle Swarm Optimization Technique

M. Fakhfakh, S. Masmoudi, Y. Cooren, M. Loulou and P. Siarry (2012). Modeling, Analysis, and Applications in Metaheuristic Computing: Advancements and Trends (pp. 154-170).

www.irma-international.org/chapter/improving-switched-current-sigmadelta/63810/ 
Pure and Hybrid Metaheuristics for the Response Time Variability Problem

Alberto García-Villoria, Albert Corominas and Rafael Pastor (2013). Meta-Heuristics

Optimization Algorithms in Engineering, Business, Economics, and Finance (pp. 275311).

www.irma-international.org/chapter/pure-hybrid-metaheuristics-response-

time/69889/ 